\title{
Developing an evaluation methodology for ICT supported learning: enhancing and promoting quality through 'change knowledge'
}

\author{
Bernadette Charlier, Joël Bonamy and Murray Saunders \\ Université de Fribourg, Université Lumière Lyon 2, University of Lancaster
}

\begin{abstract}
This paper reports on the evaluation experience in European projects focused on change in education and training. The design of evaluation processes and practices in this kind of environment provides resources for 'sense making' for those involved in the change process and in so doing re-conceptualises the notion of quality as one of enhancement and development. The paper describes the main features of the distance teaching projects evaluated, the main phases of the evaluation approach and eventually proposes a discussion on the observed effects of such an evaluation approach on quality enhancement and change processes.
\end{abstract}

Key words: Evaluation, quality, theories of change, distance learning, higher education, professional training.

\section{INTRODUCTION}

This paper reports on the evaluation experience gained in European projects focused on change in education and training. These projects were international in scope involving European countries and institutions. These experiences help to think of some of the ways in which evaluation and research into the change process involving the introduction of distant or ICT based learning support might be undertaken. The design of evaluation processes and practices in this kind of environment will provide resources for 'sense making' for those involved in the change process and in so doing re-conceptualises the notion of quality as one of enhancement and development.

The original version of this chapter was revised: The copyright line was incorrect. This has been corrected. The Erratum to this chapter is available at DOI: 10.1007/978-0-387-35700-3_33 
In summary our argument is as follows. We are working and learning in what social theory calls conditions of chronic uncertainty. These conditions can produce periods of abnormality and destructive instability. Learning changes in HE or organisations, often prompted by new ways of learning linked to the introduction of ICTs, can produce such instabilities as a transition is made across a boundary from one culture of practice to another and, for learners, from HE into new work practices. Learners and teachers or trainers and organisations can be supported by constructing provisional stabilities as they seek creative solutions to problems created by change. These provisional stabilities are produced by reflecting, learning and the understanding of change through evaluative processes, enabling choices or decisions for future action.

This paper will outline our experience of developing a methodology to undertake the provision of evaluative resources which suggests that formative evaluation can provide the resources for such reflections and act as a bridging tool for planning and innovation.

In the following sections we will first describe the main features of the distance teaching projects evaluated, then we will describe the main phases of our evaluation approach and eventually we will discuss the observed effects of such an evaluation approach on quality enhancement and change processes.

\section{INNOVATIVE DISTANCE LEARNING PROJECTS}

The distance learning projects ${ }^{1}$ evaluated share common characteristics.

\subsection{Project participants}

The projects were first of all networks of teachers or trainers, researchers and learners coming from different European institutions or organisations. While there was a common interest, professional training or teacher training, these networks were more characterised by their differences of institutional or organisational culture; of time and resources allocated to ICT education; of the university level concerned; in the main activities of the organisation, and of the languages used (French, Spanish and English).

1 JITOL for Just In Time Open Learning has been a project of the EC program DELTA (1992-1994) and was coordinated by Pr. Robert Lewis (Neurope Lab, Archamps, France and University of Lancaster, UK). LEARN-NETT for Learning Network for Teacher has been a project of SOCRATES - OPEN AND DISTANCE LEARNING EC program (1998 -2000) and was coordinated by Dr. Bernadette Charlier (University of Namur, Belgium). 
Students or trainees could be considered the main stakeholders, but these learning environments also involved academics, professors as well as research-assistants and tutors, who have developed and learned new practices during these projects and who might also be considered stakeholders.

\subsection{Project aims and activities}

The vision of collaborative learning and of its interest for professional development has formed the basis for the development of the projects. In these cases, collaboration has to be understood both as a learning strategy and as a result of learning i.e. a desired outcome. Learners are placed in a pedagogical environment in which they can interact with peers and experts inside and outside the institution. They can realise projects, share knowledge about common professional problems, experiment and reflect on the new knowledge created.

\subsection{Structure, support, tools}

The training systems developed and experimented with during the projects were supported by distance learning platforms. These platforms addressed the main dimensions of the pedagogical projects. They constituted the location and tools for the communication, collaboration and management of each group of students as well as for the group of teachers or trainers.

\section{EVALUATION ROLE}

Both projects were confronted with the introduction of new learning objectives and methods as well as with the redefinition of the roles of the actors. Both have had to redefine these main dimensions of the training system according to the analysis of the experiences of the learners, the teachers and the managers. In such circumstances, evaluation supported the quality enhancement of the project as well as the change processes by supporting knowledge building by the actors (learners, teachers, managers) on their own learning experiences as well as a better understanding of the conditions for changes in the institutions or organisation. In such circumstances, in which all the objectives, methods and tools couldn't have been known in advance, evaluation could be understood as a support for knowledge building (see in this book, Henri, Charlier, Daele and Pudelko) and regulation of the projects. This evaluation has been articulated with a 
systematic research and development process. During the project, the expression and sharing of the questions, observations and analysis was elicited to build a common frame of reference for the next investigation. At the end, the partners identified the new knowledge and questions that had arisen throughout the project, and suggested conditions for their transferability.

\section{EVALUATION PRINCIPLES}

Three key principles guide the evaluation. It should be :

- Collaborative: the aims, methods, frame of reference and planning of the evaluation are negotiated with the main actors of the projects;

- Integrated: the evaluation methodology is integrated into each of the time phases of the project and into all the different trial sites.

- Formative: the evaluation generates feedback throughout the projects.

\section{EVALUATION POSITION : EXTERNAL AND INTERNAL}

The convergence between research and development and evaluation leads to the distinction between internal and external points of view. Actors of the projects are asked to share their own goals, projects and visions of change. This process may strengthen the change process as well as the emergence of a community. Thus evaluation instruments are integrated into the systemic process of the experience in which different types of stakeholders and different institutions participate - these provide internal process-driven information.

On the other hand, the evaluation team maintains an external point of view, while benefiting from the integrated instruments. For example, it monitors open-ended questionnaires to collect data from partner members from each institution to construct individual institutional perspectives. It provides to the actors of the project regular accounts of their analysis and of their interpretative framework. This process gives the opportunity to the actors to validate the evaluation results and also contributes to the development and change process. 


\section{BUILD THE EVALUATION WORKPLAN}

Our evaluation approach leads to specific answers to the questions addressed by a generic tool used to define an evaluation workplan called RUFDATA (Saunders, 2000; Trowler, Saunders, Knight, 2002). We describe these main questions and answers below:

\section{Reasons and purposes [Why evaluate?]:}

The evaluation has two aims: to provide support to the change process (project development) and to give a deeper understanding of the innovative process and its specificities (to produce knowledge).

2. Uses [What use will it have?]: different uses are made at different stages of the evaluation process.

- Through the participation of the partners in the setting up of the evaluation workplan and tools, it will help them to integrate quality evaluation criteria into their practice during the project;

- Through the discussion and analysis of the results, the building by the partners of a better understanding of the change process experienced is supported. This is what we have called bridging tools.

\section{Focus [What will it collect evidence on?]}

In line with the democratic quality evaluation approach, the indicators of success are generated collaboratively with the participation of partners. For this stage, we use the EPO [Enabling, Process and Outcome] methodology which distinguishes between three types of evaluation indicators (Helsby and Saunders, 1993) :

- Enabling indicators are concerned with the structures and support which need to be set up or provided to support the desired processes to take place.

- Process indicators are concerned with what needs to happen within the target groups practice in order to embody or achieve desired outcomes.

- Outcome indicators are concerned with the intermediate or longer term outcomes of activities and are tied to impact goals. In the 
training systems outcomes are often related with the assessment of learning and with the institutional impact of the innovative project.

\section{Data and evidence [What kind of evidence will it use?]}

The types of data gathered are largely determined by one main common characteristic of the project evaluated: the use of ICT. ICT use enhances the opportunities to gather evidence on the learning and teaching experiences: learners' profiles through the individual web pages, learners and tutor's experience through logbooks, learning processes through the analysis of the forum and chat exchanges, quality of the knowledge building through the individual report or the analysis of the content of the databases. In addition to these data provided by the training system, the evaluation team monitored open-ended questionnaires to collect data from partner members from each institution.

\section{Audience [Who is it for?]}

Audiences of the evaluation could be differentiated with another useful tool: 'the implementation staircase' which allows the identification of the recipient of policy and the agency of policy, which could be different from one project to another. In the European projects, project coordinators are often the agents of changes with the project officers representing the European Community. Recipients are teachers, tutors, learners who could be also considered as agents of their own projects.

\section{Timing [When will it take place?]}

From our perspective, evaluation begins before the starting of the project and ends after it. At the beginning, it helps to clarify the main intentions of all the actors and the define evaluation plan. At the end, it gives time for the communication of results, the transfer of the lessons learnt at each level: learners, teachers, tutors, trainers, managers or heads of department and institutions.

\section{Agency [Who will do it?]}

As we have already emphasised, the evaluation will be carried out by the evaluation external team as well as by all the actors of the training systems. 


\section{QUALITY ENHANCEMENT AND CHANGES}

Training systems innovate by giving access to a wide variety of resources while defining new roles for the learners and for the teachers and defining new criteria for the assessment of learning. The evaluation method apprehends and articulates different dimensions. It describes and analyses the existing training program and the administrative constraints as well as the new objectives and methods and the adaptations they generate. It apprehends the whole learning and training processes and the effects on the organisational changes. It analyses also the uses of ICT as well as the participation of the actors in its design and regulations. Eventually, it leads to the expression and the sharing of the sense given by the actors to the changes experienced.

Evaluation is part of the training system itself. It has two main roles: development of the training system and production of knowledge about its design, uses and effects from the point of view of the different audiences. From this perspective, quality evaluation criteria are interpreted originally. There are not external criteria imposed from outside. The actors build these criteria themselves taking into account their diversity.

Instead of trying to reduce complexity by searching for common solutions (optimum project planning) and thus searching for a stable framework that could force change (common objectives and evaluation criteria, common academic planning, ..) we prefer to develop a process that will help each partner to participate in the design and the regulation of a common framework that will take into account his own characteristics. In other words, a framework that will create an individual provisional stability for change. The design of evaluation processes and practices in this kind of environment will provide resources for 'sense making'.

\section{CONCLUSION}

This process of sense making would help the partners of an innovative project to define specific evaluation criteria for their project and eventually to integrate them in their day to day practice. The approach described here, suitable for innovative projects, must be considered as an approach among others. Applicable to circumstances in which network of partners share a common project of innovating in training. It contributes to the development of this project.

A quality evaluation process focused on accountability must be considered as a complementary approach. Partners of the project could describe their own frame of references and answer to requests coming from 
the policy agency: i.e., the European Commission. This exchange and negotiation would be facilitated by the reification of all the criteria and tools used for the evaluation of the project.

Nowadays, HE is often confronted with the evaluation of changes in learning and teaching. One main effect that could be considered in our experience would be the strong impact of the participation of all the change agents (teachers and learners) in the evaluation process on the quality enhancement of the project and on their personal involvement.

\section{REFERENCES}

Helsby, G. and Saunders, M. (1993) Taylorism, Tylerism and performance indicators: defending the indefensible, Educational Studies, 19(1), 55-77.

Saunders,M. (2000),Beginning an evaluation with RUFDATA: theorizing a practical approach to evaluation planning, Evaluation, 6(1). 7-21.

Trowler, Saunders, Knight, (2002), A guide to change for heads of department, subject centres and others who work middle-out, Lancaster University. 